e.g. cancer. The chief advantages of the proposed methods are their speed, ease, reliability, and capacity to select individual cells from a tissue slice population with a higher degree of purity and specificity. The result is the extracted DNA can be biochemically analyzed with a higher degree of diagnostic accuracy, as the isolated sub-sample would not be diluted by unwanted material from the ambient tissue. In comparison to other techniques, the proposed methods would combine high specificity with high speed.

This work was done by Lawrence A. Wade of Caltech and Emil P. Kartalov, Darryl Shibata, and Clive Taylor of the University of Southern California for NASA's Jet Propulsion Laboratory. Further information is contained in a TSP (see page 1).

In accordance with Public Law 96-517, the contractor has elected to retain title to this invention. Inquiries concerning rights for its commercial use should be addressed to:

Innovative Technology Assets Management $J P L$

Mail Stop 202-233

4800 Oak Grove Drive

Pasadena, CA 91109-8099

E-mail: iaoffice@jpl.nasa.gov

Refer to NPO-47561, volume and number of this NASA Tech Briefs issue, and the page number.

\title{
Robotic Scaffolds for Tissue Engineering and Organ Growth
}

\section{Biocompatible and biodegradable smart scaffolds could reconfigure their shape and size to accommodate organ development.}

\section{NASA's Jet Propulsion Laboratory, Pasadena, California}

The aim of tissue engineering (TE) is to restore tissue and organ functions with minimal host rejection. TE is seen as a future solution to solve the crisis of donor organs for transplant, which faces a shortage expected only to increase in the future. In this innovation, a flexible and configurable scaffold has been conceived that mechanically stresses cells that are seeded on it, stimulating them to increased growth.

The influence of mechanical stress/ loading on cell growth has been observed on all forms of cells. For example, for cartilages, studies in animals, tissue explants, and engineered tissue scaffolds have all shown that cartilage cells (chondrocytes) modify their extracellular matrix in response to loading. The chondrocyte EMC production re- sponse to dynamics of the physical environment (in vivo cartilage development) illustrates a clear benefit (better growth) when stressed. It has been shown that static and dynamic compression regulates PRG4 biosynthesis by cartilage explants.

Mechanical tissue stimulation is beneficial and (flexible) scaffolds with movable components, which are able to induce mechanical stimulation, offer advantages over the fixed, rigid scaffold design. In addition to improved cell growth from physical/mechanical stimulation, additional benefits include the ability to increase in size while preserving shape, or changing shape.

By making scaffolds flexible, allowing relative movement between their components, adding sensing (e.g., for de- tecting response of cells to drug release and to mechanical actions), building controls for drug release and movement, and building even simple algorithms for mapping sensing to action, these structures can actually be made into biocompatible and biodegradable robots. Treating them as robots is a perspective shift that may offer advantages in the design and exploitation of these structures of the future.

This work was done by Adrian Stoica of Caltech for NASA's Jet Propulsion Laboratory. For more information, contact iaoffice@jpl.nasa.gov.

This invention is owned by NASA, and a patent application has been filed. Inquiries concerning nonexclusive or exclusive license for its commercial development should be addressed to the Patent Counsel, NASA Management Office-JPL. Refer to NPO-47142.

\section{(8) Stress-Driven Selection of Novel Phenotypes}

\section{A methodology allows the experimental design of novel peptides and RNAs that have desired properties.}

\section{Lyndon B. Johnson Space Center, Houston, Texas}

A process has been developed that can confer novel properties, such as metal resistance, to a host bacterium. This same process can also be used to produce RNAs and peptides that have novel properties, such as the ability to bind particular compounds. It is inherent in the method that the peptide or RNA will behave as expected in the target organism. Plasmid-born mini-gene libraries coding for either a population of combinatorial peptides or stable, artificial RNAs carrying random inserts are produced. These libraries, which have no bias towards any biological function, are used to transform the organism of interest and to serve as an initial source of genetic variation for stress-driven evolution.

The transformed bacteria are propagated under selective pressure in order to obtain variants with the desired properties. The process is highly distinct from in vitro methods because the variants are selected in the context of the cell while it is experiencing stress. Hence, the selected peptide or RNA will, by definition, work as expected in the target cell as the cell adapts to its presence during the selection process. Once the novel gene, which produces the sought phenotype, is obtained, it can be transferred to the main genome to increase the genetic stability in the organism. Alternatively, the cell line can be used to produce novel RNAs or peptides with selectable properties in large quantity for separate purposes. The system allows for easy, large-scale purification of the RNAs or peptide products. 